\title{
NOTAS DE TOPONIMIA ARÁBIGO-MURCIANA
}

\author{
Por \\ ROBERT POCKLINGTON
}

\begin{abstract}
Albacete
Nombre del principal heredamiento (zona de riegos) de la huerta de Lorca, que se extiende entre las ramblas de Tiata y Biznaga, y el río Guadalentín, ocupando la mayor parte del fondo de la vega. Se trata del paraje denominado al-Fundūn en las fuentes árabes, famo'so por sus altos rendimientos de cereales $v$ su sistema de riegos. Documentación: "la alqueria del Auaçete (sic)... la dicha alqueria del Aluacete" (ACL: 29-V|-1474); "el vefedor del Aluacete monde los algibes..." (ACL: 3-IX-1474); "la açequia e agua que viene al Aluaçe, por donde se riega la huerta e canpo desta çibdad» (ACL: 9-1-1490). En las Ordenanzas de 1713 hallamos la grafía Albazete (1).

El nombre parece proceder, como el de la ciudad manchega, del árabe al-Basiț "el llano" (2). Esta etimología ya se encuentra en los Blasones y Antigüedades de la Ciudad de Lorca (1741) de Fray P. Morote: «...Albacete, cuyo termino Arabigo significa tierra llana, por serlo assi toda la que riegan sus aguas" (p. 301). En los ss. XIIIXIV el nombre debió pronunciarse *Albacét, y cuando, en el $s$. XV, la -t final dejó de ser tolerada por el castellano, los lorquinos tuvieron que escoger entre la pérdida de esta - $t$ y la agregación de una -e de apoyo. La documentación demuestra que ambas soluciones coexistieron durante algún tiempo: Albacé y Albacete; la situación se repite en el topónimo - también lorquino-- de Tamarchete (s. XIII Tamarchet, Tamarchete), que en el s. XV se documenta varias veces como Tamarché (3). La conversión de la $b$ árabe en castellano medieval $\underline{y}$ era frecuente tras $/ 0 \mathrm{r}$.
\end{abstract}

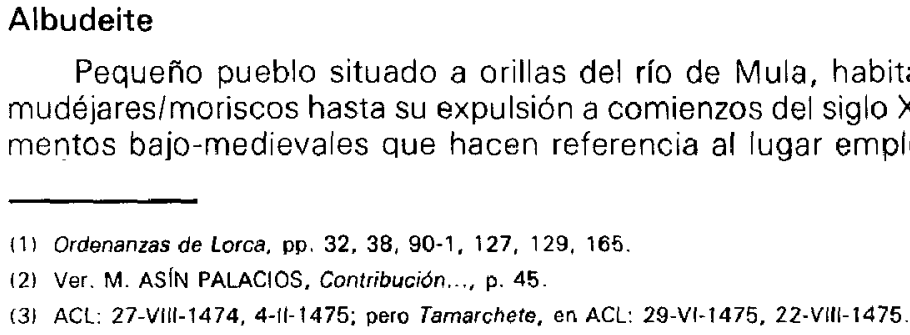

(1) Ordenanzas de Lorca, pp. 32, 38, 90-1, 127, 129, 165.

(2) Ver. M. ASÍN PALACIOS, Contribución..., p. 45.

(3) ACL: 27-VIII-1474, 4-1I-1475; pero Tamarchete, en ACL: 29-VI-1475, 22-VIII-1475.

Pequeño pueblo situado a orillas del río de Mula, habitado principalmente por mudéjares/moriscos hasta su expulsión a comienzos del siglo XVII. Los primeros documentos bajo-medievales que hacen referencia al lugar emplean ya la grafía actual: 
Albudeite (doc. de 1366) (4), Albudeyte (AC: 3-1-1380), Albudeit y Albudeite (doc. de 1447-58) (5), "el Portichuelo de Albudevte" (AMM leg. 3.076/6, n. ${ }^{\circ} 218: 1465$ ), "Juan de Ayala, señor de Albudeyte" (AC: 7-|X-1476, 25-II-1477), etc.

El primero en proponer una etimología árabe para este topónimo fue el gran arabista don Miguel Asín Palacios, quien, recogiendo la observación de Madoz según la cual el lugar «tiene un pilón de agua escasa, pero muy saludable, encañạda desde su nacimiento que está cercano y sirve para el uso del vecindario y los ganados", quiso partir del diminutivo árabe al-Buḍdayọ «el Agua Escasa" (6). Más recientemente, C. Hernández Carrasco, en su tesis doctoral sobre la toponimia murciana, aceptaría esta etimología sin reservas, aportando al mismo tiempo la imprescindible documentación medieval y un nuevo testimonio de la escasez de agua en la comarca: "...es tierra de secano con poca y mala calidad para la labranza" (s. XVIII) (7).

Sin embargo, a pesar de la aparente idoneidad de esta etimología, tiene, si la examinamos más detenidamente, dos puntos de debilidad. En primer lugar, conforme con las normas de la imela, la $u$ y a de la raíz árabe, hallándose junto a una o enfática velarizadora, debieron pronunciarse $/ 0 /$ y $/ a /$ en lugar de $/ \mathrm{u} / \mathrm{y} / \mathrm{e} / \mathrm{l} / \mathrm{al}$-boḍado $/>{ }^{*} \mathrm{Al}$ bodaite. $Y$ por otra parte, aunque nadie negaría que el agua escaseara en Albudeite, la. verdad es que en la provincia de Murcia el agua escasea prácticamente en todas partes; de hecho, encontrándose próximo a un río que siempre suele llevar algo de agua, lo suficiente para regar unos pequeños huertos, y en las cercanías de una fuente de agua saludable capaz de satisfacer las necesidades de vecinos y ganados, se podría decir que su situación respecto al abastecimiento de agua era relativamente buena.

En su Vocabulista aráuigo en letra castellana (1505), Fray Pedro de Alcalá recoge la voz bedd, pl. budŭd, con el significado de umolino de aceite». También hallamos badd como traducción del latín PRAELUS (léase PRE LUM "prensa») en el Glossarium Latino-Arabicum, compuesto en Éspaña entre los ss. VIII Y XII (p. 392). Se trata de la voz árabe de procedencia siria: badd, budūd "pressoir, grande machine servant à presser des olives ou du raisin, moulin à huile» (Dozy, Supplément, I, p. 56). El diminutivo de este vocablo constituye un étimo ideal para nuestro topónimo: al-Budayd "el Pequeño Molino de Aceite». En este caso la imela actuaría de manera inversa, dejando la $u$ con su pronunciación clásica, pero convirtiendo la a en /e/: /al-budéyd/ $>$ cast. ant. Albudeit (la $d$ final da tanto d como $t$ en el castellano murciano del s. XIV), cuya forma pronto adoptó una -e final de apoyo, una vez que la - $t$ final dejó de ser tolerada por este idioma. Es innecesario insistir sobre la importancia de los molinos de aceite, 0 almazaras, en estas tierras, donde la falta de agua hace que la aceituna sea uno de los principales productos.

\section{Algameca}

Nombre de dos pequeñas calas situadas a unos 2 kilómetros al SO de Cartagena entre la entrada del Puerto y la Isla de la Torrosa. La Algameca Chica se encuentra justamente detrás del castillo de Galeras, y en ella desemboca la Rambla de Benipila; la Algameca Grande, más ancha, está a poniente de la primera. El topónimo figura ya en un texto murciano del s. XV: "...el pescado que pescaren en Esconbrera e en el Argameca..." (AC: 7-1\}-1477); en diversos documentos de hacia 1600, C. Hernández Carrasco halló las formas Algamecas y Algameca Maor (sic por "Mayor») (8). Por

\footnotetext{
(4) C. HERNANDEZ CARRASCO, El árabe en la toponimia murciana, s. v. Albudeite, p. 186.

(5) Ibidem

(6) M. ASÍN PALACIOS, Contribución a la toponimia de España, p. 50

(7) C. HERNÁNDEZ CARRASCO, loc. cit.

(8) C. HERNÁNDEZ CARRASCO, El arabe en la toponimia murciana, pp. 190-1.
} 
otra parte, A. Merino Alvarez encontró: "Argamecas (mayor y menor) en el manuscrito de Hurtado, que se guarda en la Academia de la Historia" (2); sin embargo, no indica la fecha de este documento.

C. Hernández propone la etimología al-cAmiq "el Desfiladero Prolongado", ya que "la orografía abrupta del terreno parece confirmar este origen» (10). Pienso que esta hipótesis es, en sus rasgos esenciales, correcta. No obstante, en la forma en la que se presenta se le puede oponer algún reparo: (a) tratándose del nombre de dos calas, el significado de "desfiladero prolongado" no resulta muy apropiado; (b) sorprendería la agregación de una -a final de apoyo cuando lo normal en estas circunstancias es - $e ; y(c)$ el sonido árabe 'ayn no suele pasar al castellano como $/ \mathrm{g} /$ : partiendo de está raíz se esperaría más bien un topónimo *Alameque. Afortunadamente, estas dificultades desaparecen si tenemos en cuenta lo siguiente: $\{a\}$ el significado básico de camíq es "profundo"; (b) existe, por lo tanto, una forma femenina "amiqa "profunda", y (c) la raiz "amiq se acostumbraba pronunciar con gayn: gamiq en el árabe hispánico y magrebí (11). Reformando la etimología, ahora, a la luz de estas consideraciones, queda claro que debemos partir del hispanoárabe al-Gamiqa "la Profunda», cuya forma y significado se adaptan perfectamente a las condiciones reales del topónimo. Sólo merece comentario la pronunciación de la icomo /e/, normal en la proximidad de una q árabe velarizadora: Algameca. La conversión de la primera sílaba de la forma castellanizada Al- en Ar-, ya en nuestra documentación del s. XV, se deberá probablemente a una disimilación con la / del artículo romance: el 1/- $>$ el Ar-

\section{Arcaina o Alcaina}

Los mapas topográficos no se ponen de acuerdo en cuanto a la pronunciación de este topónimo, denominación de un pequeño valle enclavado en la vertiente occidental de la zona montuosa que se eleva entre los términos municipales de Murcia y Molina: (913c1/2) MU. Así, en el mapa de escala 1:25.000 n. ${ }^{\circ} 913-1$, recientemente editado, hallamos constantemente la variante con Al-: Casa de la Alcaina, Casa de la Alcaina Vieja, paraje de La Alcaina y Montes de Alcaina (sic), y en el $n .^{\circ}$ 913-III: La Alcayna; por otra parte, bastante más cerca de Murcia, en el lado opuesto de la sierra, a unos 3 kilómetros al $\mathrm{N}$ de Espinardo, encontramos Torre Alcayna, emplazamiento de una nueva urbanización; el paraje probablemente recibió este nombre por hallarse allí una torre, situada en el camino que se dirigía hacia La Alcayna/Alcaina. Sin embargo, en el mapa de escala 1:50.000, editado por el Instituto Geográfico y Catastral en 1933, el topónimo se transcribe La Arcaina, con la variante "Alcaina" como denominación de un vértice geodésico cercano llos nombres de los vértices geodésicos siempre han de ser tratados con recelo en los mapas de esta seriel. No dispongo de documentación antigua que pudiera indicarnos la grafía etimológica del nombre; en cualquier caso, aunque halláramos el topónimo en un texto del s. XIII no podríamos estar totalmente seguros de su valor probatorio respecto a la primacía de la grafía con Ar-o Al-, porque ya en ese siglo, tanto en el hispanoárabe murciano como en el castellano, detectamos indicios de la debilitación e inestabilidad de las $-r$ y - implosivas (12), y en cuanto esto ocurrió, el doble juego de etimología popular,

(9) Geografia Histórica de la Provincia de Murcia, p. 157.

(10) C. HERNÁNDEZ CARRASCO, log. cit.

(11) R. DOZY, Suppiément, vol. II, p. 173, y A. DiAZ GARCíA, El dialecto árabe-hispánico y el "Kităb filahn al-cămma", p. 65.

(12) En el Repartimiento de Lorca se mencionan tres pobladores "Gil de Alcayne", "Pero de Alcaynes y "Guillern de Alcayne" (pp. 37, 39), probablemente oriundos de Alcaine (Teruel), topónimo documentado como Arcayne en 1293 (vide infral. Más ejemplos infra, s. v. Bujércal. 
ante la imposibilidad de decidir si el topónimo contenía el artículo árabe Al- o la palabra Arco/Arca, provocaría una constante vacilación entre las formas Alcaina y Arcaina, llegando hasta nuestros días.

La falta de documentación antigua, junto con las consideraciones arriba expuestas, me impediría de avanzar cualquier hipótesis firme sobre el posible origen de este topónimo, si no fuera por su reaparición en otras comarcas y regiones. Me refiero especialmente al pueblo turolense de Alcaine, cuyo nombre ya fue estudiado por J. Corominas en el primer tomo de Tópica Hespérica (pp. 34-5), el cual se documenta en el año 1293 bajo la forma Arcayne, y que, según Madoz, algunos todavía llamaban Arcaine en el s. XIX. Por otra parte, con la terminación -a como el topónimo murciano, encontramos en la provincia de Almería un barranco denominado Alcayna, junto al pueblo de María (dato de Madoz recogido por Corominas), y a unos 5 kilómetros al NO de Albox los dos topónimos La Terrera de Alcaina y El Llano de Alcaina en el punto donde la Rambla de Oria sale de una zona de monte para unirse a la Rambla del Saliente: (996c1) AM. Finalmente, a 6 kilómetros al norte de la desembocadura del río Almanzora, en las inmediaciones del sitio donde la Rambla de Canalejas emerge de entre las sierras de Almagrera. El Castillarico y La Sierrecica, hallamos las Lomas de Larquín: (1.015b2) AM, forma que debe mostrar el paso de ai a $i$ por imela de segundo grado, y la aglutinación del artículo romance, si, como creemos, tiene la misma etimología.

Para el Alcaine de Teruel Corominas propuso la etimología árabe "Irq ${ }^{C} A y n$ «raíz de fuenten, o sea "lugar de emergencia de un manantial», recordando la procedencia del castellano alcazuz "regaliz" del árabe cirq al-sūs "idem", y de su sinónimo orozuz del plural árabe de la misma raíz 'curūqsūus. En cuanto al sentido del compuesto, lo compara con el aragonés antiguo raytz de la fuent "vena de la fuente», el topónimo valenciano Rossalain derivado del ár. Ra's al- ${ }^{C} A y n$ "Cabeza de la Fuente" y los varios Capafonts, Caudete, Ouibdique, etc. del latín CAPUT FONTIS o CAPUT AQUAE. En apoyo de la etimología cita a Madoz quien dice que en Alcaine, a orillas del río Martín. "brotan varios manantiales de aguas abundantes...".

No creo, sin embargo, que esta etimología sea la correcta; y eso por dos motivos. En primer lugar, porque en los demás parajes de este nombre no hallamos fuentes o manantiales; o si los hay, no son lo suficientemente importantes para figurar en los mapas. Pero sobre todo la etimología no convence porque un topónimo debe tener un significado determinado y no indeterminado, es decir, que la raíz árabe tendría que contener el artículo determinado al-: "Irq a/-`Ayn «la Raíz de la Fuente», como el nombre de formación paralela Rossalain, procedente, como se ha dicho, de $R a{ }^{\prime} s$ al- ${ }^{c} A y n$. Para salvar la hipótesis, entonces, habría que explicar la pérdida de la -1- intervocálica: "Arcalain > Arcayn(e), cosa que no creo posible, o, por otra parte, plantear el empleo en el árabe hispánico de un compuesto *cirq-cayn "raíz de fuenten, y suponer que el articulo inicial, necesario en un topónimo árabe, al menos durante su creación y consolidación en la lengua, se perdió con posterioridad: al*"Irq-"ayn > "Alarcayn > Arcayne, todo lo cual también sería muy hipotético. En vista de todas estas consideraciones, pienso que podemos abrirnos a la posibilidad de que el nombre tenga otro origen (13).

Si examinamos los emplazamientos del topónimo murciano y de los dos últimos de la provincia de Almería (desconocemos la ubicación exacta del Barranco de Alcayna en María), notamos que los tres son parajes situados junto a una rambla en el punto

(13) El prof. Muñoz Garrigós, en el X Col loqui General de la Societat d'Onomastica (Valencia, marzo 1985), propuso sacar el topónimo murciano del catalán alquena ualheñan; sin embargo, no veo ninguna manera satisfactoria, si se parte de esta raiz, de explicar el diptongo ai del nombre. 
donde ésta abandona una zona de monte o sierra, los cuales se encuentran parcialmente encerrados por los montes en cuestión. Por ello pienso que puede tratarse del árabe al-Rukayn o al-Rukayna "el Rinconcillo", origen ya propuesto por Corominas para el nombre de lugar sevillano Robaina (s. XIll Rogaena) (op: cit., p. 60). Sin embargo, bajo condiciones normales, se esperaría *Arrucaina, o mejor "Arruquena, como forma del topónimo en el castellano; para que el resultado fuera Arcaina, Alcaine, etc., serfa necesario suponer que la $u$ pretónica se pronunciaba muy débilmente, hasta el punto de no quedar reflejado en la pronunciación romance, $y$ que, como consecuencia de esta debilitación de la $u$, la rr se acercaría a la a siguiente lo suficientemente para impedir su conversión en lel, que es la pronunciación que se esperaría en una a acentuada situada entre las consonantes $k-y$. También pudieron ayudar en la fijación de la pronunciación Arcaina las analogías con la voz arco/arca y el nombre propio Caín.

Pero ¿tenemos derecho a contar con este aflojamiento o desaparición de la $u$ pretónica? La respuesta va a ser afirmativa. Se tratará, naturalmente, de un rasgo de la pronunciación árabe, pues en el castellano medieval no vamos a encontrar lo que buscamos. En primer lugar, como es sabido, la debilitación y perdida de las vocales pretónicas (kvkv́k>kkúk) es un rasgo característico del árabe magrebí actual (14). Sin embargo, no se han detectado hasta ahora los inicios de este mismo proceso en el hispanoárabe. Quiero, por lo tanto, reunir aquí una serie de ejemplos claros del fenómeno, procedentes la mayoría de la zona murciana, los cuales demostrarán que el caso de los topónimos Arcaina no es, de ninguna manera, aislado: (a) la antigua Puerta de Belchid de la ciudad de Murcia será el Bāb al-Ŷadid "La Puerta Nueva" que menciona Ibn al-Abbār (15): tras la conocida reducción haplológica $B a ̄ b$ al- > /Bel-l, la pérdida de la a pretónica daría paso a la conversión de "Belğdíd en Belchid bajo la influencia del apellido; (b) en el Repartimiento de Murcia el topónimo Rabat Algedit lár. Rabaọ al-Ŷadid "el Barrio Nuevo") a menudo se escribe Rabat Algidit; por otra parte se cita un término del Campo de Cartagena Aliadit Alffauqui lár. al-Ŷadīd al-Fawqi "lo Nuevo de Arribas); la alternación a-e-i de la a pretónica de la voz Ýadíd atestigua de nuevo su debilidad de pronunciación; (c) en distintos textos del s. XIII, una acequia de la zona de Aljucer se denomina Acequia del Lahut, de Allihud o de Alihud; se trata evidentemente del árabe al-Yahüd "los Judíos": la grafía del Lahut (=d'Ellahut) corresponde a la pronunciación clásica, con ár. -ly-> cast. "ll»; la forma Allihud indica que la a pretónica se ha convertido en lil: mientras que Alihud supone la reducción de la raíz a lal-Yhūd/ con la desaparición de la vocal pretónica; el topónimo de Soria Aliud, para el cual Asín Palacios señaló esta misma etimología (16), también remonta a /alYhüd/; en un texto murciano de 1410 se cita un "Yhuda, judio, ferrero" (17). Y el Libro del Repartimiento proporciona bastantes ejemplos más del mismo proceso, pero éste no es el lugar para enumerarlos todos. Para terminar examinaremos dos casos que revisten un interés especial: (d) la voz aduana procede, como es sabido, del árabe al-diwāna «idem» (v. DCECH s. v.); para Corominas el tratamiento de la i es anómalo, y quiere atribuir su pérdida a un desarrollo posterior dentro de las lenguas románicas que adoptan dicho arabismo; pero en vista de que esta $i$ no aparece en ninguna de las antiguas formas romances que él cita, es preferible hacer remontar su desaparición a un cambio fonético verificado dentro del árabe: se tratará de otro caso más de la debilitación o caída de la vocal pretónica lal-diwāna > al- "duwāna o al- "dwā-

(14) Ver: A. STEIGER, Contribución... pp. 86-91

(15) Para más detalles ver mi estudio: Nuevos datos sobre cinco puertas musulmanas y una torre de da cerca medieval de Murcia, at-Qantara, VI, (en prensah.

(16) M. ASIN PALACIOS, Contribución a la toponimia árabe de España, p. 64

(17) Libro de Protestaciones (AC) de 1410, f. TY (20-1X-1410), AMM 
na > romance aduana, adovana, etc.); (e) el topónimo alicantino de Guardamar que figura ya en los primeros documentos castellanos referentes a la zona, bajo la grafía actual (18); Mikel de Epalza ha propuesto, muy acertadamente, la etimología Wãdi al-Rimâl uel Río de las Arenas, o de las Dunas» (19), en vista de su emplazamiento junto a la desembocadura del Río Segura, lugar caracterizado por la presencia de importantes formaciones de dunas; el desarrollo fonético requiere la desaparición de la $i$ pretónica de la voz Rimâl y, por supuesto, la sustitución del árabe clásico Wãdi por la forma hispanoárabe Wād: Wād al-R(i)māl > * Guadarrmal (20) > Guardamar por etimología popular (Guarda-Mar) gracias a la gran movilidad de la -r implosiva en el castellano. Cfr. también el topónimo siguiente.

\section{Asnete}

En las Actas Capitulares del Concejo de Murcia de los años 1376-77, se cita en varias ocasiones un pequeño poblado de mudéjares llamado Asnete, situado en el Valle de Ricote: "en Asnete, Iugar ques del Val de Ricote" (AC: 21-VI-1376), repitiéndose el nombre en el mismo texto bajo las formas Asnet y Asnete; también hallamos Asnete en el libro correspondiente al año siguiente: AC: 8-VIII-1377.

Se trata probablemente del árabe al-Sanad "la Ladera», que iría acentuado sobre la última sílaba en el hispanoárabe (pronunciándose la a acentuada como /e/ por imela), con la pérdida de la vocal pretónica (cfr. Arcaina): las-s(a)néd/ > Asnet(e); para la adopción de la $-d$ final como $t$ compárese Albudeite. De la misma raíz proceden los topónimos Cenete (Guadix) y Sanet (Alicante y Lérida) (21), y, según ha demostrado recientemente María Jesús Rubiera (22), ciertos de los Atzeneta levantinos.

Por otra parte, aunque lo creo menos probable, no se puede excluir que el nom. bre proceda del plural Asnād "Laderas", que daría el mismo resultado fonético. En contra de esta hipótesis se puede oponer: (a) el argumento semántico de que no es muy verosímil que un caserío o aldea pequeña se construya sobre más de una ladera; y (b) se esperaría más bien "Alasnete (al-Asnād "las Laderasm) ya que los artículos casi siempre están presentes en los topónimos murcianos de procedencia árabe; cfr. sin embargo Ulea, infra.

\section{Benahendín}

Nombre de un antiguo barrio de Alguazas (pueblo situado a 14 kilómetros al NO de Murcia, cerca del punto donde el río de Mula desemboca en el Segura):

"... paresçio Pero Martines Caluillo e dixame que el, que ha una casa en el regno de Murçia que disen Alguasa de Cotiellas i Benahendyn» (doc. de 1319 recogido en otro de 1382) (23).

La expresión "Alguasa de Cotiellas" se emplea para distinguir este "Alguasa» de otro lugar homónimo ubicado en la zona sur de la Huerta de Murcia; el pueblo de Cotillas (hay Torres de Cotillas) se encuentra a unos 2 kilómetros al sur de Alguazas. El topónimo que nos interesa se repite dos veces más en la misma carta real, escrito una vez Benahendyn y la otra Benahandyn. También figura, bastante borrado, en un

\footnotetext{
(18) CODOM I, p. 59: 1271; CODOM H, pp. 87-8: 1288, y pp. 110-116: 1295; atcétera.

(19) M. DE EPALZA, Al topónimo de Guardamar, esp. pp. $207-9$.

(20) Como en el caso de Arcaina, la proximidad de la $\mathrm{rr}$ a la a de la sílaba siguiente impide su conversión en lel por imela en el entorno consonantico $\mathrm{m}-1$.

121) M. ASIN PALACIOS, Contribución a la toponimia árabe de España, pp. 102, 132-3

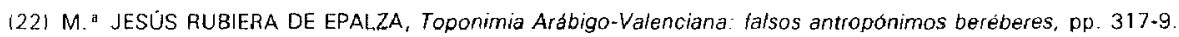

.(23) AMM Cartulario Real 1384-91, ff. $28 \mathrm{r}$ y $28 \mathrm{v}$.
} 
documento de 1354 que hace referencia a las aljamas de una serie de poblaciones y lugarejos murcianos:

"... a los alcaldes e alguazil de Murçia e de Lorca e de los Alguaşças e del Alcantariella e de Cotiellas e de Be.h. din e de Ceuti $\theta$ a qualquier..." (24).

No he hallado referencia a este lugar en ningún otro texto.

La etimología del nombre deja poco lugar a dudas: se tratará del árabe Binā' Hamdīn "la Casa de Hamdinn». Este nombre propio no es muy frecuente en las fuentes hispanoárabes, pero Ibn al-Abbār biografía a un Hamdīn b. Muḥammad b. 'Ali...b. Hamdin al-Taglabī, cordobés, cuya familia era oriunda de un pueblo de Granada (25), y menciona también a Abū ${ }^{\mathrm{C}} \mathrm{Abd}$ al-lāh b. Hamdīn en otra biografía (26); por otra parte, Ibn 'Arabī de Murcia, en su Epístola de la santidad, hace referencia a una obra de otro cadí cordobés llamado Abū al-Qāsim b. Hamdīn (27). A pesar del parecido formal, no creo que pueda existir ninguna relación con el nombre del pueblo granadino de Alhendín, en árabe al-Hamdãn (28), pues la conversión de à acentuada en /i/ por imela de segundo grado, tan corriente en el árabe granadino tardío, no se daba en el árabe murciano; Hamdān habria dado *Hendén.

\section{Beniaçam}

Antigua alquería del término de Orihuela, cuyo nombre estudiamos aquí porque, al estar ubicada muy cerca de la frontera con Murcia, se cita en ciertos documentos murcianos de los ss. XIII-XIV que son decisivos para aclarar su etimología. Aparece el topónimo por primera vez en el Libro del Repartimiento de Murcia, grafiado Benayçam y Benyçam, constando, además, que se hallaba cerca de Zeneta (Murcia):

«Et dieronle camio en Benyçam, alcaria de Orihola, en lo de Gombalt de Cançer. I ataffullas" (Rept. Murc., p. 238)

"... diemos a uos, Catalina de Calders, ...xx ataffullas en Oriuella, en Azenet, en lo que fue de Gonbalt de Cançer, en linde de Domingo Tomen (Ibídem).

«...et diemoslas a Domingo Tome, adalil, por camio de las $\mathrm{xx}$ ataffullas que auie en Benayçam que le tomamos, que diemos a Catalina de Caldes" (Op. cit., p. 244).

«... a uos, Domingo Tome, adalil, por emienda de las $x x$ ataffullas que auiedes en Benaycam que diemos a Catalina de Caldes" (Op. cit., p. 245).

En el año 1320 se celebró un pleito entre Murcia y Orihuela, suscitado por los perjuicios que ocasionaba a ciertos vecinos de Murcia con heredades en Beniel, Raa! y Alfandarín, una presa nuevamente construida por los oriolanos en el Segura, junto a la frontera con Murcia:

«...por razon de fazimiento i del desfazimiento de la presa que fue fecha de nueuo en el rio de Segura, en termino de Orihuela, çerca Beniaçam, a tiniente del termino de Murçia, para regar las heredades de Moquita i de Beniaçam, terminos de Orihuela, por la qual los herederos de Beniaffel, de Raffal i d'Alfandari, terminos de Murçia, dizen que reçibian danyo en sus heredades i en sus anyoras que tienen d'antigo fechas en los dichos lugares" (29).

\footnotetext{
(24) AMM Cart. Real 1348-54, 1. 89v: este documento está editado en CODOM VIl, p. 145, pero incorrectamente interpretado el topónimo.

(25) Takmila, biog. n. 119

(26) Takmila, biog. n. 81

(27) M. ASIN PALACIOS, Vidas de Santones Andaluces, p. 172.

(28) M. ASIN PALACIOS, Contribución a la toponimia árabe de España, p. 64

(29) AMM Pergamino Original n. ${ }^{\circ} 95,6-V \| 1320$. Vid. también s. v. Beniçamed, infra
} 
De este texto se desprende que la alquería de Beniaçam se encontraba junto a la frontera con Murcia, y si recordamos que el Repartimiento la sitúa "en Azenet", debía estar en el lado sur del río, por lo que cabría ubicarla en el paraje de Las Norias, o hacia Los Desamparados, por donde aún pasa la Acequia de Moquita.

Las diversas grafías que se conservan del topónimo demuestran que se trata del

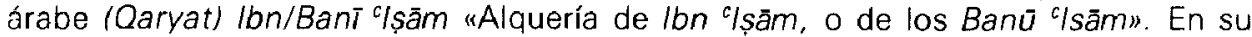
Qașida Maqșūra, al-Qarțāŷannī menciona un lugar llamado Bani 'cșām (verso 464), y su comentarista Abū ał-Qāsim Muḥammad b. Aḥmad al-Garnāți lo sitúa en Orihuela (30): será, con seguridad, el mismo sitio. Estas tierras indudablemente pertenece-

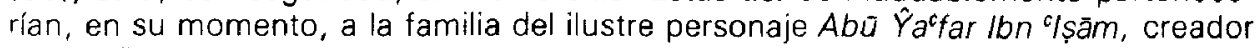
de la Wizāra "șāmiyya o consejo de hombres sabios que gobernó el estado independiente de Orihuela entre los años de 1239-1266 (31). Por otra parte, el nombre bajo el cual el pueblo de Raal figura en el Libro del Repartimiento: Raffal Abenayçam, del árabe Raḥal lbn c/șām "Cortijo de Ibn "șām», demuestra que antes de la Reconquista también pertenecería a un miembro de esta familia (32).

En lo que se refiere al desarrollo fonético, para las formas Benyçam y Benayçam del Libro del Repartimiento hay que partir de la raiz lon $\% / s a a m$, pues lbn se pronunciaba normalmente /(A)bén)/ en el árabe hispánico, y la combinación ci a menudo pasa al castellano como /ay/ o /ey/. Así, por ejemplo, el topónimo Rahal Aben Cabayn (Rept. Murc., p. 249) será el árabe Rahal Ibn Sabcin "Cortijo de Ibn Sabinn, y la alquería de Benieça (ár. Bani '`sà) a veces figura como Benieyça o Benayça. Por otra parte, la forma Beniaçam del s. XIV procederá del étimo citado por al-Qarțâyannī: Banī "șām, pasando por la pronunciación intermedia *Beni-ayçam, perdiéndose la -y implosiva a causa de la debilidad de su posición y quizás también por disimilación con la otra $i$.

\section{Beniçamed}

Nombre de una presa o azud, situada en el río Segura, en término de Orihuela, cerca de la frontera de Murcia y del paraje de Beniaçam cuyo nombre acabamos de estudiar. El topónimo se menciona en el texto de un acuerdo concluido entre los concejos de Murcia y Orihuela en 1416 sobre la trayectoria del Azarbe de Monteagudo, el cual recogía aguas muertas de las tierras bajas situadas junto a Monteagudo para verterlas en en el río. Este azarbe necesitaba atravesar una parte del término de Orihuela para poder desaguar en el Segura porque enfrente de Monteagudo la ribera del río estaba más alto que las aguas estancadas del almarjal; sólo llevando el agua bastantes kilómetros en dirección a Orihuela, en un azarbe (cauce artificial para transportar aguas sobrantes), resultaba posible ganar altura con respecto al río, para que all se pudiera verter en él. Sin embargo, a los oriolanos les estorbaba este cauce. y decidieron obstruirlo con un banco de tierra junto a la frontera con Murcia, construyendo un azarbe nuevo que condujera el agua directamente de este punto al río:

"... antigamente se solian escorrer las aguas del almarjal que disen de Montagudo en el açarbe viejo, el qual entraua i escurre e vasia en el rio de Segura çerca del adarbe de la dicha villa de Orihuela... I ... abrieron et fisieron otro açarbe nueuo por donde se escurriesen las aguas del dicho almarjal que engrana en el dicho rio en termino de Beniaçam, huerta de la dicha villa [de Orihuela]..." (AC: 19-VII-1416).

(30) Kitab rafc al-ḥuýub al-mastura fi mahasin al-maqsüra, imprenta al-Sacåda, El Cairo, 1925, vol, 1, p. 176

(31) E. MOLINA LOPEZ, El circulo político-literario de la "Wizära 'Ișámivyán de Orihuela (s. KIIII. Revista Azahara n. "9, oct. 1980. Murcia, pp. 64.71

(32) Tambiên en el Campo de Cartagena había, en el s. Xill, un rahal denominado Rahal Aben Levçam (Rept. Murc., p. 249), probablemente Rahilibn al-c/sam; sin embargo, en vista de la presencia del articulo en el nombre, cabe sospechar que se trata de un personaje distinto. 
Pero esta solución dejaba insatisfechos a los murcianos, pues reducía la eficacia del azarbe. Para que el agua entrase en el río en este lugar era preciso elevar su nivel con una parada en el azarbe; esta obstrucción, al detener el agua, empantanaría las zonas más bajas. Al final se acordó:

«que era mas prouechoso... el açarbe nueuo, que non el viejo, alargandose el dicho açarbe nueuo dessde la puente de la Cañal (sic) fasta debaxo de la Açud de Beniçamed, que es en la huerta i termino de la dicha villa [de Orihuela]" (loc. cit.).

Se tratará del árabe Bani Șamad o Sāmid "Descendientes de Samad o Sāmid". nombres propios que significan "firme, ininmutable», que, sin embargo, no he hallado hasta ahora en las fuentes biográficas hispanoárabes. Por otra parte, no pademos excluir que proceda de Bani Şāal-amit "los descendientes de al-Sāmit» cuyo nombre quiere decir "el Silencioso", ya que este étimo daría el mismo resultado fonético (-t $y-d$ finales se intercambian en el castellano medievall; se cita un personaje llamado 'Ubāda b. al-Şāmit en el Musnad de Ibn Marzūq (33). El topónimo ibicenco Beniçàmid tendrá probablemente el mismo origen (34).

\section{Benizar}

Aldea serrana situada a unos 13 kilómetros al NO de Moratalla, dominada por un castillo y enclavada en un pequeño valle junto a la frontera de la provincia de Albacete. El topónimo se menciona ya en uno de los primeros documentos castellanos referentes al reino de Murcia, en el cual el Infante don Alfonso, el futuro rey Alforiso $X$ el Sabio, confirma la donación de una larga lista de castillos de la Sierra de Segura a la orden de Santiago: "...Muratalla, Socouos, ...Lietor, Aznar, Abeneyçar, Nerpe, Tayviella...) (doc. de 1243) (35). Posteriormente, en dos documentos de 1273 y 1274 hallamos la forma Benevcar (36). C. Hernández Carrasco, en su estudio del topónimo (37), indica que aparece escrito Abeneyzar en el Bullarium de la Orden de Santiago, publicado por J. López Arguleta en 1719; se tratará probablemente de una copia de nuestro primer documento de 1243, donde el amanuense (o el editor) ha alterado o "corregido" la grafía original. Por otra parte, la forma Beniçar que la misma investigadora halló en las Actas Capitulares del Concejo de Murcia (23-XI-1493) no corresponde a este topónimo, sino a la Acequia de Benizá, cuarto tramo de la Acequia Mayor del Norte (Llano de Brujas-Santa Cruz) que desciende por la orilla izquierda del río Segura, aguas abajo de la ciudad de Murcia.

De nuevo, M. Asín Palacios fue el primero en proponer una etimología árabe para este topónimo: Bani Zahr, nombre de familia (38). No obstante, como ya señaló C. Hernández en su citado estudio, esta etimología es incompatible con la documentación antigua del nombre, de la que no disponía el ilustre arabista al redactar su libro: el diptongo ley/ queda inexplicada, y resultaría extraño que la $z$ sonora árabe diera consistentemente una "ç" sorda castellana launque es preciso reconocer que la grafía "Ç» no se distingue rigurosamente de la «z" en los textos del S. XIII); en todo caso, el elemento Aben- de la primera.forma documentada demuestra que hay que partir de un étimo con Ibn $\vee$ no Banit.

\footnotetext{
(33) M. J. VIGUERA, El Musnad: hechas memorables de Abül-Hasan, sultán de los Benimerines, Inst. Hispano-árabe de Cultura, Madrid, 1977, p. 523, n. $^{\circ} 726$.

(34) J. COROMINAS, Entre dos Ilenguatges, Curial, Barcelona, 1976, tomo II, p. 223.

(35) CODOM III, p. 4

(36) CODOM II, pp. 50 \& 54.

1371 C. HERNANDEZ CARRASCO, El arabe en la toponimia murciana, pp. 163-4.

1381 M. ASIN PALACIOS, Contribución..., p. 93.
} 
C. Hernández Carrasco, teniendo como principal punto de referencia la forma

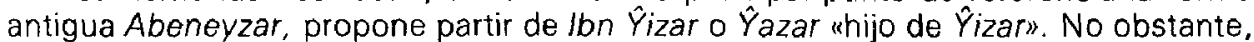
esta hipótesis sigue sin explicar el diptongo /ey/: de esta raíz se esperaría "Abengizar, "Abenzar o, en el mejor de los casos, "Abenizar pero no Abeneyzar. Y cuando recordamos que las auténticas grafías antiguas son Abeneyçar y Beneyçar, las cuales nos son conocidas gracias a la cuidada publicación de los documentos originales por el Dr. Torres Fontes en sus CODOM II y CODOM III, encontramos también que ni la $\hat{y}$, ni la $z$ de esta raíz árabe pueden dar (satisfactoriamente) una "ç" castellana.

Entonces, ajustándonos estrictamente a las primeras formas documentadas, pienso que hay que partir de un nombre propio árabe Ibn Aysar, por Ibn al-Aysar «hijo del Zurdon. El apodo de al-Aysar fue llevado notablemente por Muhammad IX, sultán del reino de Granada (39); y por otra parte en un documento arábigo-granadino de 1495 se cita a una señora, hija de Anmad al-Aysar (40). La ausencia o pérdida del artículo al-en antropónimos de este tipo parece haber sido bastante común en el árabe local: así, por ejemplo, el topónimo huertano de Benialé (s. XIII Benihalel) procede del nombre de una familia murciana Bani al-Halläl, algunos de cuyos miembros fueron biografiados por Ibn al-Abbār (41); en el Campo de.Cartagena existia un rahal denominado Beniatim (ár. Ibn/Bani Yatīm), y otro de Galib Aliatin (ár. Gălib al-Yatīm) (42); y como ya vimos s.v. Beniaçam, se encuentra cerca del río Segura un Raffal Abenayçam (Rahal Ibn 'Ișām) v, al mismo tiempo, en el Campo de Cartagena Rahal Aben Leyçam (Rahạal Ibn al-' Ișām).

\section{Bujércal}

Paraje de secano situado junto a la via férrea entre Lorca y Baza, a unos 12 kilómetros al sur de la ciudad de Lorca. El topónimo se documenta desde la época de la Reconquista: "...afruenta con el Açequia del Pozo et con el aluar faza Buxercal" (Rept. Lorca, p. 47; 1265-1330). En el s. XV se menciona un charco de este nombre: "el Charcol de Buxercal" (ACL: 24-XI-1489 y 27-111-1490); en la segunda cita la -I final de Charcol está tachada; supongo que se pronunciaría Chárcol con una -1 final antietimológica (43), bajo la influencia de la - / final de Buxércal, dando al topónimo completo un cierto equilibrio rítmico: Chárcol de Buxércal. Por otra parte, en su Historia de los Riegos de Lorca (1847), D. J. Musso y Fontes incluye entre los cauces correspondientes al heredamiento de Albacete (vide supra, s.v.) el Real Canal de Bujércal (p. 135).

Parece tratarse de la kunya árabe Abū Sākir («Padre de Sākir»), llevada por diversos personajes hispanoárabes biografiados en la Takmila de Ibn al-Abbār; p. ej. Abü Sāakir 'Abd al-Wāhid b. Mawhab (n. $\left.{ }^{\circ} 28\right)$ y Abū Šăkir Hāmid b. Hāmid de Mallorca (n. ${ }^{\circ} 96$ ). El desarrollo fonético del topónimo presenta alguna complejidad, pero resulta verosímil si se tienen en cuenta ciertos rasgos del dialecto árabe murciano, o de la transmisión de los arabismos en esta comarca. En primer lugar encontramos indicios de una tendencia de convertir las raíces del tipo KăKiK en KăKaK: por ejemplo, la voz wäriț "heredero" siempre da "Huarat» o "Huarad" en el Repartimiento de Murcia; así,

\footnotetext{
(39) L. SECO DE LUCENA, Muhammad ix, Sultan de Granada, Patronalo de la Alhambra, Granada, 1978, p. 256

140) L. SECO DE LUCENA, Documentos Arábigo-Granadinos, pp. 145-6.

(41) Takmila, núms. 174 y 251

1421 Rept. Murc., op. 248-9
}

143) La agregación de 10 -r implosivas, especialmente en posición final, tiene sus raices en la debilitación general de las consonantes implasivas y la ultracorrección de esta tendencia: se detecta en varios topónimos lorquinos a partir de estas fechas, p. ej. la Capellanía de Almohaxar (Libro de Aguas, n. ${ }^{\circ}$ g, f. 11 r. 1583 ) remonta al apellido Almohaja del Rept. de Lorca, y el topónimo Viotar (974 b 4$)$ AM procede del apellido Biota del misma texto. 
no sería extraño que Saakir pasara a "Sāakar. Y segundo, en bastantes ocasiones encontramos una $r$ implosiva antietimológica en antropónimos musulmanes y topónimos árabes de origen antroponímico: p. ej. en el Libro del Repartimiento, al lado de los nombres propios Ahmad Açaheli, Juçeff Açahelli (pp. 193, 195), derivados de la nisba árabe al-Sahli "él del Llano», encontramos el topónimo (Rahal) Azarhelli (p. 248); en la página 228 aparece el nombre propio Alauargiri al lado de Alauagiri, tratándose del árabe al-Lawâyiri "el Rajolero, Fabricante de Ladrillos»; en el topónimo Rahal Erça Amnalhaig (p. 250) el segundo elemento será el nombre propio árabe ${ }^{\top} \bar{T}$ sà "Jesús", que suele dar Eça en este texto; y el antropónimo Caçim Aben Margcohl (p. 230) será Qāsim Ibn Makhül (44); etc. Este segundo rasgo también podría ser atribuido a la pronunciación de los conquistadores castellanos o catalanes, ya que hallamos algún caso en formas no procedentes del árabe; por ejemplo el poblador Guillem de Narbona se denomina de Narbonar en la página 220 del Repartimiento de Murciá; sin embargo, existe abundante evidencia de una gradual debilitación de las consonantes implosivas en el hispanoárabe, una de cuyas consecuencias podría ser el desarrollo de estas $-r$ antietimológicas. Pero, cualquiera que sea el origen del fenómeno, está claro que pudo afectar el nombre *'Săkar (pron. /šékar/). Entonces IAbú Sé(r)kar/ daría Buxercal tras una disimilación $r-r>r-1$, y pérdida de la $A$ átona de $A b \bar{u}$, resultados habituales en topónimos de estas características (45).

\section{Javalí}

Nombre, en la actualidad, de dos poblaciones situadas en el extremo NO de la Huerta de Murcia: Javall Viejo, sobre la orilla izquierda del río, y Javalí Nuevo, de más reciente construcción, en la ribera derecha. El topónimo aparece por primera vez en un texto anterior a 1322 (en copia de hacia 1400), donde se trata, por supuesto, de Javalí Viejo: «El açarbe mayor de parte del Açequia Aljeufia, deuen fazer mondar todas las otras de Beniajam (46) fasta El Jaualin (47). En otro pasaje del mismo libro de Ordenanzas Antiguas, fechado en 1353 e intitulado Partiçion del agua del Açequia de Aljufia, se lee: "Primeramente, asinamos El Jaualin, segunt que la auia et devia aver, media fila corrible» (58). C. Hernández halló, por estos mismos años, las grafías Gaualin y Jaualin (49), y las menciones del paraje se hacen frecuentes en las décadas siguientes: "los escoredores (sic) ençima de los Arcos et del Jaualin» (1364) (50): "la heredat que disen del Gaualin... auia mester faser... en la açequia mayor una añora" (AC: 22-XII-1399): "una aldea que disen del Jaualyn» (AC: 13-III-1408). A partir de estas fechas empieza a ser frecuente la grafía sin -n final: El Jaualy (51), alternándose con la forma más antigua El Jaualin (52). La existencia de Javall Nuevo está atestiguada ya en un documento de 1594, en el cual el lugar primitivo de Javalí se denomina, lógicamente, Javali Viejo (53).

\footnotetext{
(44) Cfr. Makhül al-Sămī, quien, utransmitió directamente de Mahoman (v. M. J. VIGUERA, Musnad, p. 507, N. ${ }^{0} 550$.

(45) Un argumento a favor del origen romance de la -r intrusiva seria que, de estar va presente en la raiz árabe, habria impedido la pronunciación de la à como /e/, por lo que tendríamos "Buxárcal. Pero ello dependeria de la capacidad de reacción de la imela en esta época. ¿Era un fenómeno aún vivo, continuamente ajustando la pronunciación de las vocales a su entorno consonántico? 0 , por el contrạrio, ¿estaba ya marcada desde antiguo la pronunciación de cada vocal, variándose en épocas tardias solamente la intensidad de la alteración de las vocales?

(46) Creo que debe leerse Beniaçam, refiriéndose a Raal (s. XIII Raffal Abenayçam); Beniajam, hoy Beniaján, está en el otro lado deł río.

(47) J. TORRES FONTES, El regadio murciano..., p. 42.

(48) Op. cit., p. 55

(49) El árabe en la toponimia murciana, pp. 223-4: docs. de 1325 y 1341.

(50) J. TORRES FONTES, op, ait., p. 58

(51) AC: 25-VI-1413, 24-lif-1416, 14-X-1421,4-V-1476, 2-X-1481, etcétera.

(52) AC: $10-\mid X-1421,4-X-1421,13-V I I-1473,28-1-1477$, etcétera

153) A. MERINO ALVAREZ, Geografía bistórica.... p. 241
} 
La idea de derivar este topónimo del árabe $\hat{Y a b a l}$ Ayl "Monte del (sic) Ciervo" fue avanzada por J. Vallvé en su importante estudio sobre la Cora de Tudmir, en el cual señala que los autores hispanoárabes nombran, entre los lugares de recreo de Murcia: al-Rišăa, al-Zanaqăt y Ŷabal Ayl (54). Sus fuentes debieron ser Abū al-Fidā' (Taqwīm al-Buldãn) y al-Oalqašandĩ (Şubh al-Aç̌à), en ambos de cuyos textos se hallan los mismos tres topónimos en el mismo orden lel segundo autor tomo abundantes datos del primerol, con la indicación de que eran todos lugares de recreo, 0 paseos, en Murcia. El tercer topónimo figura en ambos textos grafiado $\hat{\gamma} a b a l / y /(55)$, pero teniendo en cuenta las características de la escritura árabe, se puede leer perfectamente: Ýabal Ayl.

Sin embargo, esta etimología no es aceptable para Javall por dos motivos. En primer lugar, Javali Viejo se encuentra en un llano, lejos de cualquier montaña. $Y$ segundo L. Seco de Lucena indudablemente acierta al identificar el topónimo Ayl/IVI con el lugar de Ayăo que al-Qarțâyanni describe como "total de la cortesía", y que, por hallarse en la documentación castellana medieval como Ayelo, Xierra de Yelo, podemos situar en Verdolay, al pie de la Sierra de la Cresta del Gallo, entre Algezares y La Alberca. La grafía árabe Ayl//yl, vocalizada adecuadamente, también puede leerse Ayálo.

La etimología de los dos topónimos Javali es, por lo tanto, bien sencilla, siendo, con algunas matizaciones y a pesar de las dudas expresadas por $\mathrm{C}$. Hernández, la propuesta por M. Asín Palacios: Yabali «Montaraz" (56). En vista de que la población antigua no se encontraba próxima a una zona montuosa, es preciso optar por el origen antroponímico, partiendo de la nisba al-Ŷabali (57) «el Montaraz, el Montesino,

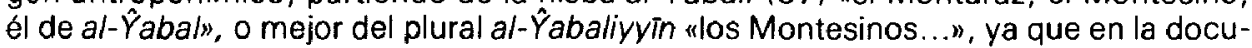
mentación más antigua predomina la terminación en -ín. Sorprende, por otra parte, el paso del árabe $b$ al castellano antiguo $u$, y la conversión de $\mathrm{Al}$ - en $\mathrm{El}$, ya que en los arabismos del murciano medieval la $b$ árabe suele dar una $b$ castellana y el artículo árabe acostumbra conservarse como Al-. Verosímilmente ambos cambios pueden ser achacados a la influencia de la voz jaball "puerco salvajen, que en los SS. XIV-XV se escribía (y pronunciaba) javalí ( $v$. DCECH s.v.); la misma influencia explicaría la pérdida de la $-n$ final a partir del $s$. XV.

\section{Ulea}

Dos topónimos: un pueblo ubicado sobre la ribera izquierda del río Segura, a la altura del valle de Ricote (912 a 5) MU; y un paraje con una ermita y arroyo del mismo nombre, situado junto a la carretera Moratalla-Calasparra $(\mathrm{km} .8)$, al sur del río de Moratalla ( $890 \mathrm{~d} 2$ ) MU. En el curso de sus investigaciones toponímicas, C. Hernández Carrasco encontró el primer lugar documentado como "Peñas de Olea» (1447581, y en los Anales de Orihuela (ss. XIV-XVI) como Ulea (58).

Siguiendo a A. Steiger (59), propuso derivar el nombre del pueblo del árabe "Uliyya o "Ulliyya (sic) "la Alta". Sin embargo, estas raíces no darían el deseado

\footnotetext{
(54) J. VALLVE BERMEJO, La Cora de Tudmi, p. 176.

(55) Ver: C. HERNANDEZ CARRASCO. El árabe en la toponimia murciana, p. 223, y al Qalqašandi. Subh al-Aç̧a..., traducción de L. Seco de Lucena, p. 41 y esp. nota 76.

(56) M. ASIN PALACIOS, Contribución..., p. 113.

(57) Nisba llevada por uno de los discipulos de Baqī b. Majlad: Abü `Abd al-Lăh Muhammad b. Ahmad al-Y̌abali (v. Manuela Marín, Baqi b. Majlad..., o. 1966 l. Cfr. tambien el predio mallorquino de Gebelli (s. XIII aAlgebelin), ASÍN PALACIOS, Op cit., p. 107

(58) C. HERNẢNDEZ CARRASCO, El árabe en la toponimia murciana, pp. 233-4

(59) A. STEIGER, Toponimia árabe de Murcia, p. 21
} 
resultado fonético porque la $T$ acentuada no pasaría a /el, y sólo podriamos esperar * Olía (60). Para que el resultado sea Olea (la primera forma documentada) hay que partir de un diminutivo "Ulavya "la Altica" (61), o acaso de la voz "Alaya "Lugar Elevado" (Dicc. Corriente), cuya $A$ pudo sonar $10 /$ a causa de la 'ayn precedente; en cualquiera de los dos étimos la a tónica intermedia se pronunciaría normalmente /el por imela. Es algo sorprendente, por otra parte, la ausencia del artículo árabe, tan frecuente en los topónimos árabes murcianos; pero teniendo en cuenta que los pueblos cercanos de Ceutí y Lorquí también han perdido los suyos, no cabe insistir excesivamente sobre este detalle.

\title{
BIBLIOGRAFIA Y ABREVIATURAS
}

\author{
AC: Actas Capitulares del Concejo de Murcia (AMM). \\ ACL: Actas Capitulares del Concejo de Lorca (Archivo Municipal de Lorca). \\ AMM: Archivo Municipal de Murcia.
}

ASIN PALACIOS, M. Contribución a la toponimia árabe de España, Madrid-Granada, 1940.

- Vidas de Santones Andaluces, Hiperión, 57 12. ${ }^{8}$ ed.), Madrid, 1981.

CODOM, vide TORRES FONTES Y MOLINA MOLINA

COROMINAS, J., Tópica Hespérica, 2 vols., Gredos, Estudios y Ensayas 169, Madrid 1972

COROMINAS, J., y PASCUAL, J. A., Diccionario crítico etimológico castellano a hispánico (DCECH), Madrid, 1980-(?). DCECH, vide COROMINAS Y PASCUAL.

DIAZ GARClA, A. El dialecto árabe-hispánico y el "Kitâb fîlahn al-'âmma” de lbn Hišâm al-Lajmí, Tesis doctorales de la Universidad de Granada 56, Univ. de Granada, 1973.

DOZY, R., Supplément aux dictionnaires arabes, 2 tomos, Leiden 1881 (Reimpresión, Beirut, 1968).

EPALZA, M. DE, Al topónimo de Guardamar, Rev. del Instituto de Estudios Alicantinos, n. ${ }^{\circ} 29$, Alicante 1980, pp. 205-214. GLOSSARIUM, Glossarium latino-arabicum; ed. preparada por C. F. Seybold, Berlín 1900.

HERNÁNDEZ CARRASCO, C., El árabe en la toponimia murciana, Anales de la Universidad de Murcia, Vol. XXXIV (1978), pp. 153-257.

MARÍN, M., Baqi b. Majlad, Al-Qantara I (1980), pp. 165-208.

MERINo ALVAREZ, A., Geografia histórica de la Provinciá de Murcia, Acad. Alfonso X el Sabio, Murcia, 1978 (1. "ed., Madrid, 1915 .

MOLINA MOLINA, A. L., Documentos de Pedro / (CODOM VII), Murcia 1978

MOROTE, P., Blasones y antigüedades de la ciudad de Lorca, Murcia 1741 (Reimpresión de la Agrupación Cultural Lorquina, Lorca, 1980\%.

MUSSO Y FONTES, D. J., Historia de los riegos de Lorca, Murcia, 1847 /Reimpresión de la Agrupación Cultural Lokquina, Lorca 1982 - Cl. Juan XXIII, 4, Lorcal.

ORDENANZAS DE LORCA, Ordenanzas y privilegios de la muy noble y leal ciudad de Lorca, Granada, 1713 (Reimpresión de la Academia Alfonso $X$ el Sabio, Murcia, 1983 ).

POCKLINGTON, R., Nuevos datos sobre cinco puertas musulmanas y una torre de la cerca medieval de Murcia, Al-Qanțara VI (1985) (en prensa)

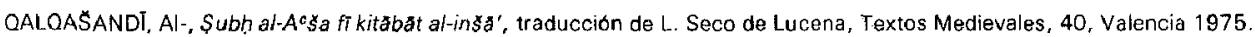
REPT. LORCA, vide TORRES FONTES.

REPT. MURC, vide TORRES FONTES.

1601 Cfr. Olias (Málaga), del árabe culiyva «Altura» según M. ASIN PALACIOS, Contribución..., p. 126.

$161)$ Cfr: los topónimos actuales: Camino del Altillo (913 a 6 ) AL. Altico de Abajo (Caserio 1910 d 6) MU. Casas del Altet $(870$ d 4) AL, Cortijo del Altico (889 c 4) MU, etcétera. 
RUBIERA DE EPALZA, M. J., Toponimia arábigo-valenciana: falsos antroponimos beréberes, Miscel.lània Sanchis Guarner, I. Universitat de València, 1984, pp. 317-20.

SECO DE LUCENA, L., Documentos arábigo-granadinos, Inst. de Estudios Islámicos, Madrid, 1961.

STElGER, A., Contribución a la fonética del hispanoárabe..., R. F. E. (Anejo XVIII, Madrid, 1932.

- Toponimia árabe de Murcia, Acad. Alfonso X el Sabio, Murcia, 1958 Iprimero apareció en Murgetana, n. ${ }^{\circ} 11$, Murcia, 19581.

TAKMILA, Complementum Libri Assilah de Aben al-Abbar, Bibl. Arábico-Hispana, tomos V-VI, Madrid, 1889.

TORRES FONTES, J., Repartimiento de Murcia, Academia Alfonso X el Sabio, Madrid, 1960.

- Repartimiento de Lorca, Ayuntamiento de LorcalAcad. Alfonso X el Sabio, Murcia, 1977.

- Documentos de Alfonso X el Sabio (CODOM I), Murcia, 1969

- Documentos del siglo XIII (CODOM II), Murcia, 1969.

- Fueros y privilegios de Alfonso X el Sabio al reino de Murcia (CODOM III), Murcia 1973

- El regadío murciano en la primera mitad del siglo XIV, Junta de Hacendados de la Huerta de Murcia, 1975.

VALLVÉ BERMEJO, J., La Cora de Tudmir, Al-Andalus, vol. XXXVII (fasc. 1), 1972, pp. 145-189.

VIGUERA, M. J.. El Musnad: hechos memorables de Abú I-Hasan. Sultán de los Benimerines, Inst. Hispano-árabe de Cultura, Madrid, 1977.

\section{MAPAS DEL INSTITUTO GEOGRÁFICO NACIONAL}

La ubicación de muchos topónimos se indica con referencia a los mapas de 1:50.000 del Instituto Geogrático Nacional, al dar, en primer lugar, el número del mapa, y luego la situación del topónimo dentro del mapa mediante una cuadrícula de $8 \times 5$ casillas, numeradas del 1 al 8 horizontalmente, y de a a e de arriba a abajo. Así, un topónimo siłuado en el extremo noroeste del mapa recibirá la referencia "a $1 ", y$ uno situado en el centro "c4» 0 "c5"; luego dos mayúsculas indican la provincia $(\mathrm{AL}=$ Alicante, $\mathrm{MU}=$ Murcia, $\mathrm{AM}=$ Almeria, etc.$\}$; por ejemplo, (934a2) $\mathrm{MU}$ es la referencia para la ciudad de Murcia 